\title{
Intralayer coupling in self-organized Fe nanoclusters grown on vicinal $\mathrm{Si}(111)$
}

\author{
M. Cougo dos Santos, J. Geshev, L. G. Pereira, M. C. M. Alves, and J. E. Schmidt \\ Instituto de Física-UFRGS, 91501-970 Porto Alegre, RS, Brazil \\ P. Allongue \\ Laboratoire de Physique de la Matière Condensée (CNRS UMR 7643), Ecole Polytechnique, 91128 Palaiseau, France
}

(Received 12 November 2003; revised manuscript received 19 March 2004; published 29 September 2004)

\begin{abstract}
This paper reports on the magnetic properties of Fe thin film grown on vicinal $\mathrm{Si}(111)$. The surface analysis, performed via scanning tunneling microscopy, showed that the iron grows in a form of two distinct types of elongated nanosized grains, aligned parallel and perpendicular to the substrate steps, respectively. A phenomenological model was used to interpret the experimental magnetization data which considers the two types of Fe nanoclusters with both cubic and uniaxial anisotropies, where those of each type are all identical and aligned. Every particle of the first type is coupled to the adjacent particle of the other type only via direct exchange coupling through the corresponding surface atoms. The experimental hysteresis loops as well as the coercivity and remanent magnetization angular variations are very well reproduced by the model, demonstrating that the magnetic behavior of this system is mainly determined by the ferromagnetic intralayer exchange coupling.
\end{abstract}

DOI: $10.1103 /$ PhysRevB.70.104420

PACS number(s): 75.30.Gw, 75.60.-d, 68.55.-a, 68.37.-d

\section{INTRODUCTION}

Studies on magnetic thin films have shown a strong correlation between the substrate's surface and the morphology of the magnetic layers, whose properties can be significantly modified as compared to those in the respective bulk materials. It has been shown that, in addition to the magnetocrystalline anisotropy, a uniaxial magnetic anisotropy is induced when the film is either obtained by means of obliqueincidence deposition ${ }^{1-4}$ or grown on a stepped surface, ${ }^{5-7}$ even in the absence of magnetic field. In the case of iron thin films, this induced anisotropy can favor magnetic moment alignment perpendicular to the steps ${ }^{7}$ as well as parallel to the step edges. ${ }^{8}$

Model calculations based on single-phase models have successfully reproduced inverted hysteresis loops measured for Fe films on $\mathrm{Si}(111)$ considering vicinal surfaces and competing anisotropies. ${ }^{9}$ Gester et al. ${ }^{10}$ have also explained the magnetic behavior in their $\mathrm{Fe} / \mathrm{GaAs}$ films by coexistence of cubic and uniaxial anisotropies. Systems consisting of two distinct magnetic phases show different behaviors, suggesting antiferromagnetic interstripe coupling of dipolar origin ${ }^{11}$ in the case of double-layered nanostripes of $\mathrm{Fe}$ on vicinal $\mathrm{W}(110)$ showing out-of-plane anisotropy. A ferromagnetic coupling of magnetostatic type is found instead for stripes with in-plane easy axis. ${ }^{12}$ Antiferromagnetic coupling of nonmagnetostatic type (indirect exchange coupling) has been observed in parallel Fe nanostripes on a vicinal W(110) covered by $\mathrm{Au} .{ }^{13}$ The negative differential magnetization when switching between magnetically saturated states in ultrathin Fe films grown on stepped W(100) has been explained by theoretical simulations that incorporates antiferromagnetic exchange coupling between nanodomains. ${ }^{14}$ Valvidares et $a l .{ }^{15}$ showed that, for small angle between the individual magnetizations, the anisotropy of two ferromagnetically exchange-coupled uniaxial anisotropy layers can be substituted by competing twofold and fourfold anisotropies acting on the average magnetization of the sample.
Direct (interfacial) exchange coupling is found to play an important role in materials with nanograin structure (see, e.g., the recent Skomski's review on nanomagnetics ${ }^{16}$ ). It is well known that in the case of high-remanent magnets, the effect of the exchange coupling between the magnetic grains leads to a significant enhancement of the remanence and energy product as compared to the decoupled magnet. ${ }^{17-22}$ Clemente et al. proposed a model for this enhancement which requires the magnetic grains to be in intimate contact in order to maximize the ferromagnetic exchange interaction between adjacent grains. ${ }^{23}$

In this paper we report experimental and theoretical results for a single $3 \mathrm{~nm}$ thick Fe layer grown on vicinal $\mathrm{Si}(111)$. The origin of the anisotropies involved, as well as the dependence on the Fe thickness of the morphological and magnetic evolutions of the film, will be discussed in more detail in a forthcoming work. Here, our attention will be focused on the complex magnetic properties of the film and on the model adopted to explain them.

\section{RESULTS AND DISCUSSION}

\section{A. Sample preparation}

The substrate we used was $n$-type $\operatorname{Si}(111)$ wafer with a miscut angle of $0.5^{\circ}$ towards the $[11 \overline{2}]$ direction as estimated by conventional $\mathrm{x}$-ray diffractometry. The substrate cleaning procedure used here is very similar to the one described in Refs. 24-26. Initially, the wafer was cleaned in two volumes of sulfuric acid and one volume of $30 \%$ by weight aqueous hydrogen peroxide for $20 \mathrm{~min}$ at $100{ }^{\circ} \mathrm{C}$. It was rinsed with $18 \mathrm{M} \Omega \mathrm{cm}$ water between and after each cleaning step. The clean wafer was then immersed and held vertically in $40 \%$ $\mathrm{NH}_{4} \mathrm{~F}$ contained in Teflon vials, and sparged with argon for $20 \mathrm{~min}$. The $3 \mathrm{~nm}$ thick iron film was deposited at room temperature by rf sputtering in $3.2 \times 10^{-3}$ Torr argon atmo- 

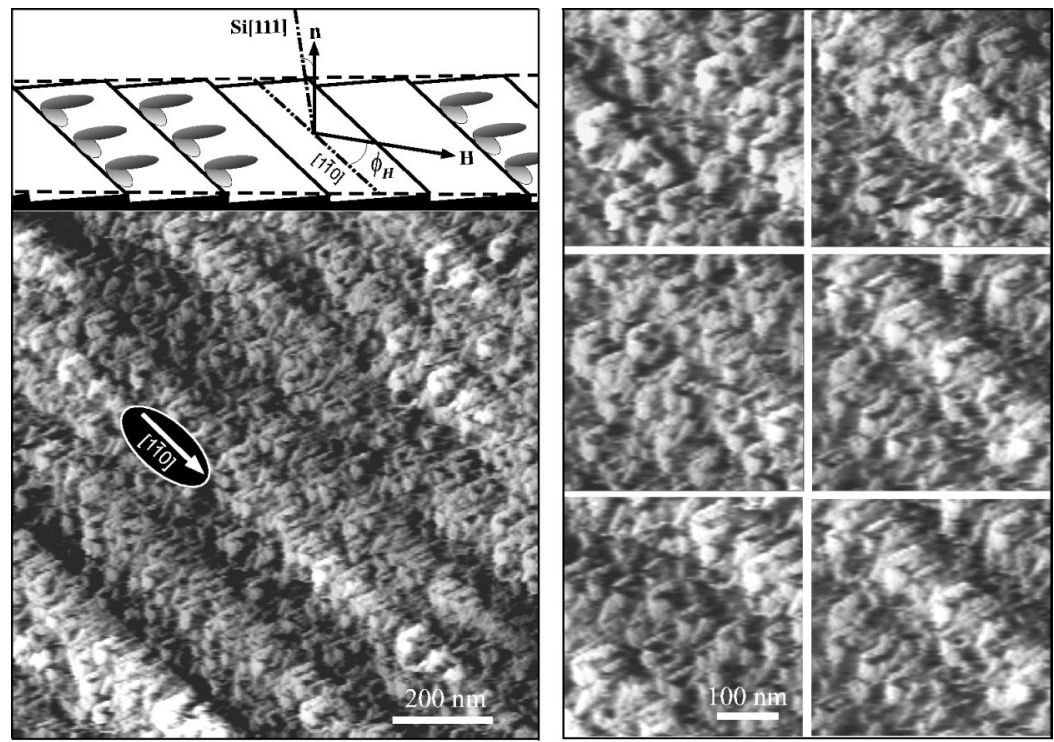

FIG. 1. Left panel: $1 \mu \mathrm{m} \times 1 \mu \mathrm{m}$ STM image of the $3 \mathrm{~nm}$ thick Fe film as well as a schematic perspective view of the grains oriented in-plane parallelly and perpendicularly to the steps. Right panel: $0.35 \mu \mathrm{m} \times 0.35 \mu \mathrm{m}$ STM images of six different areas of the film's surface. sphere with base pressure before depositing better than $10^{-7}$ Torr , and deposition rate of $0.2 \AA / s$.

\section{B. Structural characterization}

In addition to the conventional x-ray diffractometry, which indicated (111)-textured Fe, the structural characterization was performed by x-ray absorption spectroscopy (XAS) measurements as well as via scanning tunneling microscopy (STM) using a Digital Instruments Nanoscope IIIa.

The $0.5^{\circ}$ tilt of the substrate from the (111) plane produced approximately $1.8 \mathrm{~nm}$ high bunched steps with a spacing of about $180 \mathrm{~nm}$. Figure 1 shows a STM topography images of the film, whose morphology is characterized by self-organized elongated Fe nanoclusters, grown on the substrate's steps, the latter being clearly visible in the left panel of the figure as well. About half of the grains (with mean length of $70 \mathrm{~nm}$ and standard deviation of $5 \mathrm{~nm}$ ) are aligned along the $[1 \overline{1} 0]$ direction, i.e., parallel to the steps. The rest of these grains (with a mean length of $50 \mathrm{~nm}$ and and the same $\sigma$ as the others) are oriented in plane along a direction perpendicular to the steps, and are slightly larger than the others. A schematic perspective view of this structure is also shown in Fig. 1.

XAS measurements at the Fe $K$ edge were performed at the National Synchrotron Light Laboratory-LNLS, using the XAS beam line. ${ }^{27}$ A "channel cut" Si(111) crystal was used. The monochromator was calibrated at the Fe $K$ edge using a Fe metal foil. The spectrum of the $3 \mathrm{~nm}$ thick Fe film was recorded at room temperature in fluorescence mode using a $\mathrm{Ge} 15$ detector and the standard compounds ( $\mathrm{Fe}$ and $\mathrm{Co}$ metal) in transmission. The sample was placed at $45^{\circ}$ with respect to the incident beam. The spectra were collected with a point frequency of $0.8 \mathrm{eV}$ in the near edge region [x-ray absorption near edge structure (XANES)] and $2 \mathrm{eV}$ in the extended $\mathrm{x}$-ray absorption fine structure (EXAFS) region. The WINXAS program ${ }^{28}$ was used for the data analysis. The EXAFS signal between 3 and $13.6 \AA^{-1}$ was Fourier transformed with a $k^{2}$ weighting and a Kaiser window ( $k$ is the wave vector). Structural parameters were obtained from least squares fitting using experimental phase shift and amplitude functions extracted from a Co metal foil.

In Fig. 2 the Fe $K$ edge XANES spectra for the $3 \mathrm{~nm}$ thick $\mathrm{Fe}$ film and for standard $\mathrm{Fe}$ are presented. The similarity of the XANES structures in the film and in bulk Fe demonstrates that the Fe structure in the film is bcc. The smoothening in the XANES features [Fig. 2(a)] points to a higher disorder (thermal and static) in the film. The Fourier transforms (FT's) of the EXAFS oscillations [Fig. 2(b)] recorded for the $3 \mathrm{~nm}$ thick Fe film exhibit peak positions similar to that for bulk Fe. In fact, the quantitative analysis of the first peak of the FT for the $3 \mathrm{~nm}$ thick Fe film yields crystallo-
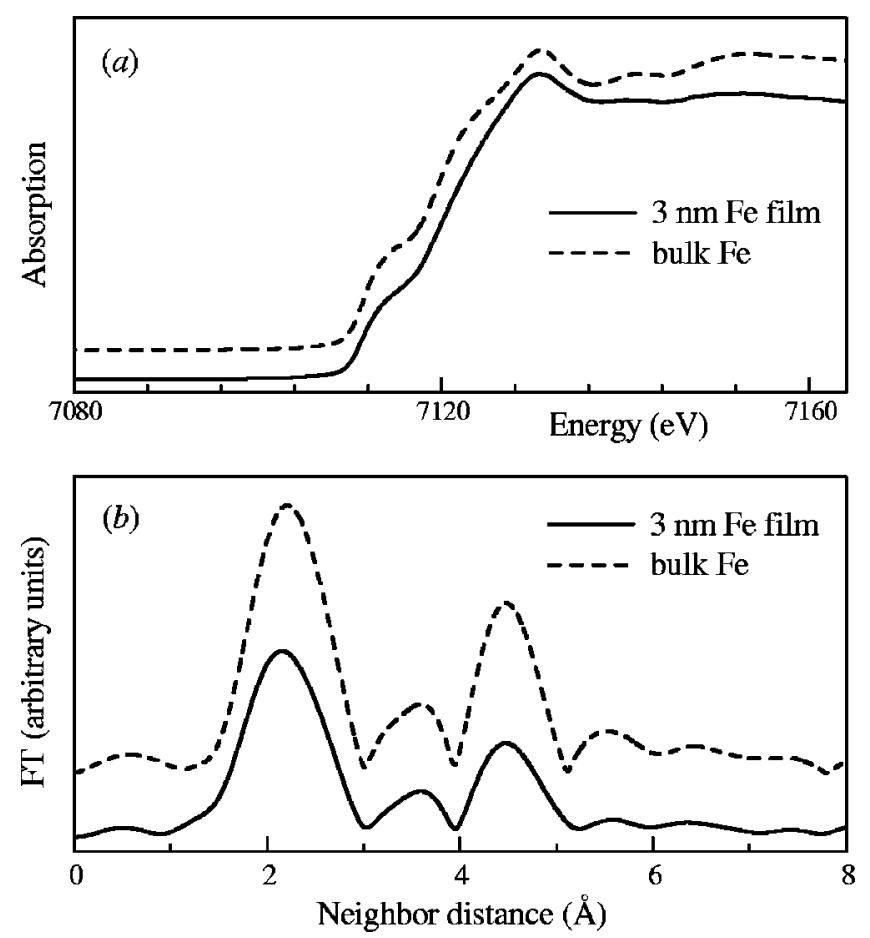

FIG. 2. (a) XANES spectra recorded at the Fe $K$ edge; (b) Fourier transform of Fe $K$ edge EXAFS signal. 
graphic parameters which correspond to bulk Fe. The XAS results, although obtained at room temperature and after the magnetic characterization, demonstrate the formation of bcc $\mathrm{Fe}$ and no trace of oxide or silicide formation.

\section{Magnetic characterization}

The magnetic characteristics of the sample were measured at room temperature and in ambient air via a homemade magneto-optical Kerr effect magnetometer. The direction of the magnetic field $\mathbf{H}$ was varied in the sample's plane (here the field angle $\phi_{H}$ equals to zero for $\mathbf{H}$ along the [1 $\left.\overline{1} 0\right]$ direction).

In order to understand the physics behind the magnetic behavior of the sample, a phenomenological free energy model was used. To prevent misleading interpretations based on this approach, however, some basic rules of thumb were followed.

It is known that if one uses a large number of fitting parameters, it is possible to reproduce well, in practice, any particular experimental hysteresis loop. So, the number of these parameters should be kept to a minimum. Afterward, as much parameters as possible should be related to values that are already accepted by the scientific community as standards (extracted from published data, such as lattice parameters, anisotropy constants of known structures, etc.), and the validity of their application should be evaluated for any particular case (which is the one of nanostructures in the present work).

A strong validation of true representation of the physics associated to the parameter set is thus obtained if one applies the same set to adjust a systematic series of different data curves (of the same system), that were measured varying an extensive parameter such as, e.g., the in-plane angle of the applied field in relation to a fixed reference line on the sample's surface. Hence, a series of 36 experimental hysteresis loops, measured along different in-plane directions, were successfully fitted with only one minimal set of parameters. Moreover, an excellent agreement between the experimental and the calculated angular dependences of the coercivity $H_{c}$ and the remanent magnetization $M_{r}$ was found.

Representative hysteresis loops (symbols) for six magnetic field angles are plotted in Fig. 3. There, the lines are the corresponding calculated curves obtained using the model described below. As can be seen, the type of the anisotropy cannot be determined from these loops since, excluding the one for $\phi_{H}=0^{\circ}$ and the peculiarity in the shape at $90^{\circ}$, the other loops are rather similar. More information can be obtained from Fig. 4, which shows the angular dependence of the measured $H_{c}$ and $M_{r} / M_{s}$ (where $M_{s}$ is the saturation magnetization).

At first sight, it seems that the competition between uniaxial and cubic anisotropies determines the observed behavior since $H_{c}\left(\phi_{H}\right)$ for $\phi_{H} \in\left(30^{\circ}, 150^{\circ}\right)$ has almost identical shape to the one for pure sixfold anisotropy (not shown), and the deep minima at $\phi_{H}=0^{\circ}$ and $180^{\circ}$ could be attributed to a superposition by a uniaxial anisotropy with axis parallel to the steps. If so, however, the maxima at $\phi_{H}=90^{\circ}$ and $270^{\circ}$ should be much higher than those observed; also, the exis-

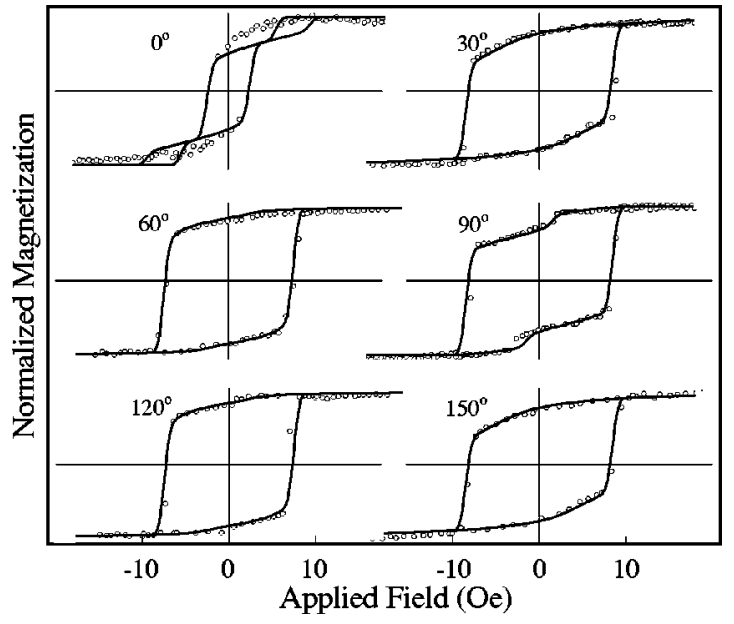

FIG. 3. In-plane hysteresis loop data (symbols) and the corresponding calculated curves (lines) for six representative magnetic field angles; the parameters used are $V_{B} / V_{A}=1.5, H_{1}^{i}=170 \mathrm{Oe}, H_{2}^{i}$ $=36 \mathrm{Oe}, H_{u}^{A}=9 \mathrm{Oe}, H_{u}^{B}=13.5 \mathrm{Oe}, H_{d, i}^{\mathrm{eff}}=18.7 \mathrm{kOe}$, and $H_{E}=3.8 \mathrm{Oe}$, whose definitions are given in the text.

tence of the sharp maxima at the vicinity of $180^{\circ}$, for example, cannot be explained by no means using such an argument. All our attempts to reproduce the measured $H_{c}\left(\phi_{H}\right)$ and $M_{r}\left(\phi_{H}\right)$ variations, assuming two independent magnetic phases, each characterized by cubic magnetocrystalline and in-plane uniaxial anisotropies with any orientations and strengths, have failed. Recently, Fu et al. ${ }^{29}$ observed very similar shape for the coercivity angular variation in their Per-

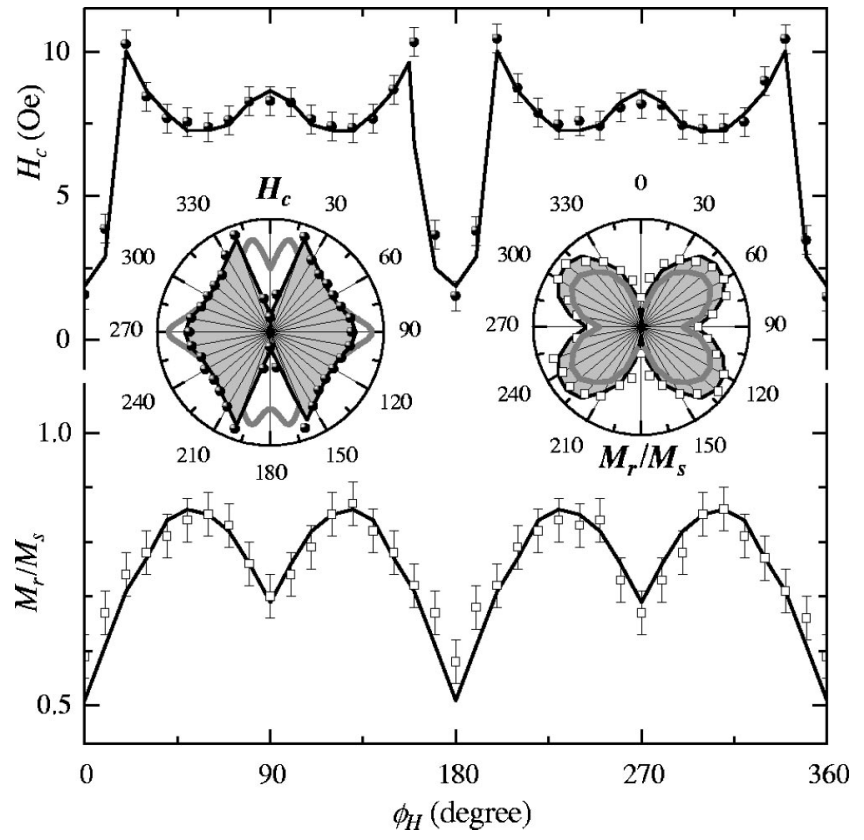

FIG. 4. Angular dependences of the experimentally measured coercivity, normalized remanent magnetization (symbols), and the corresponding calculated variations (lines). The fitting parameters used in the calculations are the same as those given in Fig. 3. The insets show the polar plots of the same variations, where the gray lines correspond to model hysteresis loops calculated using $H_{E}$ $=1.9$ Oe instead of 3.8 Oe. 
malloy film on Mo stepped surface, and also did not succeed to fit it considering coexisting cubic and uniaxial anisotropies.

Based on the STM images and on XAS atomic structure information, as well as on the discussion above, we adopted the following model. Let us consider the Fe film as a magnetically heterogeneous system composed of two parts, denoted as $A$ and $B$, consisting of elongated magnetic particles whose anisotropy has both cubic magnetocrystalline and uniaxial components. The particles in each part are all identical and aligned, with magnetization vectors $\mathbf{M}_{s}^{i}$ (where $M_{s}^{i}=M_{s}, i=A$ or $B$ ), characterized by the direction cosines $\alpha_{1, i}, \alpha_{2, i}$, and $\alpha_{3, i}$, referred to the cube axes, and volumes $V_{i}$, respectively. Every $A$ particle is coupled to the adjacent $B$ particle only (via direct exchange coupling) through the contact area $\sigma$. For fixed $\mathbf{H}$, neglecting the thermal activation effects and considering only coherent magnetization rotation, the anisotropic part of the total free energy of this system is

$$
E=V_{A} E_{A}+V_{B} E_{B}+\sigma E_{\text {int }} .
$$

The energies involved in $E_{i}$ are of the form

$$
\begin{aligned}
E_{i}= & K_{1}^{i}\left(\alpha_{1, i}^{2} \alpha_{2, i}^{2}+\alpha_{2, i}^{2} \alpha_{3, i}^{2}+\alpha_{3, i}^{2} \alpha_{1, i}^{2}\right) \\
& +K_{2}^{i} \alpha_{1, i}^{2} \alpha_{2, i}^{2} \alpha_{3, i}^{2}-K_{u}^{i}\left(\mathbf{M}_{s}^{i} \cdot \hat{\mathbf{u}_{i}} / M_{s}\right)^{2} \\
& -K_{d, i}^{\mathrm{eff}}\left(\mathbf{M}_{s}^{i} \cdot \hat{\mathbf{n}} / M_{s}\right)^{2}-\mathbf{M}_{s}^{i} \cdot \mathbf{H},
\end{aligned}
$$

where $K_{1}^{i}$ and $K_{2}^{i}$ are the first two cubic anisotropy constants, $K_{u}^{i}$ the uniaxial ones, and the last two terms refer to the effective demagnetization energies (with constants $K_{d, i}^{\text {eff }}$ ) and the Zeeman energy terms, respectively. The unit vectors $\hat{\mathbf{u}}_{i}$ and $\hat{\mathbf{n}}$ represent the directions of the corresponding uniaxial anisotropy axes and the normal-to-the-film direction. The last term in Eq. (1), $\sigma E_{\text {int }}$, where

$$
E_{\mathrm{int}}=-J_{E} \mathbf{M}_{s}^{A} \cdot \mathbf{M}_{s}^{B} / M_{s}^{2}
$$

is the exchange coupling one. As the particles $A$ and $B$ are in direct contact, they are exchange coupled via the corresponding surface atoms, and this interaction is represented by the effective coupling constant $J_{E}$ (positive). As seen, the coupling term is directly proportional to the surface contact area, and the influence of the intergrain exchange coupling predominates as the ratio of the surface area to volume $\sigma / V_{i}$ increases with decreasing the grain size. The negative sign in the above expression corresponds to ferromagnetic interactions.

As seen, the expression for $E_{\text {int }}$ does not include a term that accounts for the dipolar $A-B$ interparticle interactions. Actually, we have examined such a possibility, considering a dipolar energy of a form

$$
E_{\mathrm{dip}}=\frac{\mathbf{M}_{s}^{A} \cdot \mathbf{M}_{s}^{B}-3\left(\mathbf{M}_{s}^{A} \cdot \hat{\mathbf{r}}\right)\left(\mathbf{M}_{s}^{B} \cdot \hat{\mathbf{r}}\right)}{R^{3}},
$$

where $\hat{\mathbf{r}}$ is a unit vector along the direction that connects the magnetic moments $\mathbf{M}_{s}^{A}$ and $\mathbf{M}_{s}^{B}$, and $R$ is the distance between them. Taking into account the above energy term, however, led to calculated (using a wide range of possible combinations of the other parameters) coercivity and remanence angular dependences very different from the experi- mentally measured ones. It is most likely that the net dipolar field, if one considers the effect of all neighboring particles, is negligible. Thus, the dipolar energy term has been neglected in the present model. The four-variable minimization procedure used here for the hysteresis loop calculations was established in our previous works. ${ }^{9,30}$

The lines in Figs. 3 and 4 correspond to the calculations. As can be seen, all experimental hysteresis loops (except, to some extend, the one for $\phi_{H}=0^{\circ}$ ) as well as the $H_{c}$ and $M_{r}$ angular variations are very well reproduced by the model. The small asymmetry in the model $H_{c}\left(\phi_{H}\right)$ is due to the tilt from the (111) plane. Such an asymmetry has been observed by $\mathrm{Fu}$ et al. $^{29}$ as well. Four nonequivalent maxima in the $M_{r}\left(\phi_{H}\right)$ have also been observed (although not explained) in $\mathrm{Fe}$ on $\mathrm{Si}(111)$ films. ${ }^{31}$

The effective fields used the calculations are the cubic anisotropy fields $H_{1}^{i}=2 K_{1}^{i} / M_{s}$ and $H_{2}^{i}=2 K_{2}^{i} / M_{s}$, the uniaxial anisotropy ones $H_{u}^{i}=2 K_{u}^{i} / M_{s}$, the effective demagnetization fields (which contain any surface anisotropy contribution) $H_{d, i}^{\text {eff }}=4 \pi M_{s}^{\text {eff }}$, and the exchange coupling field $H_{E}$ $=\sigma J_{E} /\left(V_{A} M_{s}\right)$. For magnetization rotation in the (111) plane, the cubic anisotropy is given by $K_{2}$ only. In the case of vicinal surface, however, the $K_{1}$ must be taken into account in Eq. (2) as well. The $0.5^{\circ}$ tilt from the (111) plane, obtained by the conventional $\mathrm{x}$-ray diffractometry, has been provided by taking the polar and the azimuthal angles of $\hat{\mathbf{n}}$ to be $55.2^{\circ}$ and $45^{\circ}$, respectively. We also took $\hat{\mathbf{u}_{A}}\left\|[1 \overline{1} 0], \hat{\mathbf{u}_{B}}\right\|[11 \overline{2}]$, and $V_{B} / V_{A}=1.5$, in compliance with the STM topography images. The best results have been obtained for $H_{1}^{i}=170 \mathrm{Oe}$, $H_{2}^{i}=36 \mathrm{Oe}, H_{d, i}^{\mathrm{eff}}=18.7 \mathrm{kOe}, H_{u}^{A}=9 \mathrm{Oe}, H_{u}^{B}=13.5 \mathrm{Oe}$, and $H_{E}=3.8$ Oe. The difference between $H_{u}^{A}$ and $H_{u}^{B}$ can be attributed to the more elongated shape of the clusters which are perpendicular to the steps.

At first glance, the number of parameters used seems to be rather large. However, although $H_{1}^{i}$ and $H_{2}^{i}$ influence the general shape and the mean values of the coercivity and the remanence dependences, deviations of even $15 \%$ from these parameters do not significantly modify the fine details of the shape of these curves. The same holds for $H_{d, i}^{\text {eff }}$ : values $15 \%$ higher or lower than that used here do not change significantly the shape of the curves. Moreover, the $H_{1}^{i}$ and $H_{d, i}^{\text {eff }}$ values are in a very good agreement with those obtained by Gester et al. ${ }^{10}$ for Fe films of similar thickness.

The fine adjustment of the $H_{c}$ and $M_{r}$ angular variations, which was one of the challenging tasks of the present study, has been actually done by changing $H_{u}^{i}$ and $H_{E}$ only. We obtained that variations of $H_{u}^{A}$ and $H_{u}^{B}$ strongly influence the values of the coercivity and the remanence in the global minimum and local maximum regions, i.e., $\phi_{H} \approx 180^{\circ}$ and $90^{\circ}$, respectively. For example, $15 \%$ deviations from the best fitting parameter values led to variations of $\approx 50 \%$ in the corresponding $H_{c}$ in these regions.

It is worth noting that although the $H_{E}$ value of $3.8 \mathrm{Oe}$ is rather small as compared to the other effective fields, taking into account the exchange interactions is of crucial importance for the fittings. (This value is low mainly because $H_{E}$ is proportional to the contact area, the latter being very small in the present case.) Decreasing the $H_{E}$ value to $1.9 \mathrm{Oe}$, for example, the "butterflylike" polar plots of $H_{c}$ and $M_{r}$ change 
considerably (the gray lines in the insets in Fig. 4), thus demonstrating the strong influence of the intralayer coupling on the magnetic behavior of the sample.

In summary, STM topography images of our 3-nm-thick Fe film deposited on vicinal $\operatorname{Si}(111)$ showed that the iron grows in a form of two distinct types of self-assembled mutually perpendicular elongated nanoclusters. A phenomenological model, which incorporates competing cubic and uniaxial anisotropies as well as interparticle interactions was used to reproduce the experimental magnetization data. It considers that every particle of one type is directly exchange coupled to the adjacent particle of the other type only. The excellent agreement between theory and experiment indi- cates that the ferromagnetic intralayer exchange coupling greatly determines the magnetic behavior of this system.

\section{ACKNOWLEDGMENTS}

The authors thank Dr. S. R. Teixeira for assistance with the conventional $\mathrm{X}$-ray characterization as well as to the staff of LNLS for operating the storage ring and the assistance during the experiments at the XAS beamline. The beam time was supported by LNLS under the Project No. XAS1-1595. This work has also been supported by CNPq, FAPERGS, CAPES/COFECUB, and CAPES/PROCAD.
${ }^{1}$ T. G. Knorr and R. W. Hoffman, Phys. Rev. 113, 1039 (1959).

${ }^{2}$ D. O. Smith, J. Appl. Phys. 30, 264S (1959).

${ }^{3}$ J. R. Childress, R. Kergoat, O. Durant, J.-M. George, P. Galtier, J. Miltat, and A. Schuhl, J. Magn. Magn. Mater. 130, 13 (1994).

${ }^{4}$ Y. Park, E. E. Fullerton, and S. D. Bader, Appl. Phys. Lett. 66, 2140 (1995).

${ }^{5}$ B. Hillebrands, P. Baumgart, and G. Güntherodt, Phys. Rev. B 36, 2450 (1987).

${ }^{6}$ A. Berger, U. Linke, and H. P. Oepen, Phys. Rev. Lett. 68, 839 (1992).

${ }^{7}$ J. Chen and J. L. Erskine, Phys. Rev. Lett. 68, 1212 (1992).

${ }^{8}$ R. K. Kawakami, E. J. Escorcia-Aparicio, and Z. Q. Qiu, Phys. Rev. Lett. 77, 2570 (1996).

${ }^{9}$ M. Cougo dos Santos, J. Geshev, J. E. Schmidt, S. R. Teixeira, and L. G. Pereira, Phys. Rev. B 61, 1311 (2000).

${ }^{10}$ M. Gester, C. Dadoo, R. J. Hicken, S. J. Gray, A. Ercole, and J. A. C. Bland, J. Appl. Phys. 80, 347 (1996).

${ }^{11}$ J. Hauschild, U. Gradmann, and H. J. Elmers, Appl. Phys. Lett. 72, 3211 (1998).

${ }^{12}$ J. Hauschild, H. J. Elmers, and U. Gradmann, Phys. Rev. B 57, R677 (1998)

${ }^{13}$ M. Pratzer and H. J. Elmers, Phys. Rev. B 66, 033402 (2002).

${ }^{14}$ Y. Yao, H. C. Mireles, J. Liu, Q. Niu, and J. L. Erskine, Phys. Rev. B 67, 174409 (2003).

${ }^{15}$ S. M. Valvidares, L. M. Álvarez-Prado, J. I. Martín, and J. M. Alameda, Phys. Rev. B 64, 134423 (2001).

${ }^{16}$ R. Skomski, J. Phys.: Condens. Matter 15, R841 (2003).

${ }^{17}$ X. C. Kou, M. Dahlgren, R. Grössinger, and G. Wiesinger, J. Appl. Phys. 81, 4428 (1997).

${ }^{18}$ D. Goll, M. Seeger, and H. Kronmüller, J. Magn. Magn. Mater.
185, 49 (1998).

${ }^{19}$ R. Fischer, T. Leineweber, and H. Kronmüller, Phys. Rev. B 57, 10723 (1998).

${ }^{20}$ H. W. Zhang, Z. G. Sun, S. Y. Zhang, B. S. Han, B. G. Shen, I. C. Tung, and T. S. Chin, Phys. Rev. B 60, 64 (1999).

${ }^{21}$ B. Z. Cui and M. J. O'Shea, J. Magn. Magn. Mater. 256, 348 (2003).

${ }^{22}$ H. W. Zhang, S. Y. Zhang, B. G. Shen, and H. Kronmüller, J. Magn. Magn. Mater. 260, 352 (2003).

${ }^{23}$ G. B. Clemente, J. E. Keem, and J. P. Bradley, J. Appl. Phys. 64, 5299 (1988).

${ }^{24}$ K. Itaya, R. Sugawara, Y. Morita, and H. Tokumoto, Appl. Phys. Lett. 60, 2534 (1992).

${ }^{25}$ C. P. Wade and C. E. D. Chidsey, Appl. Phys. Lett. 71, 1679 (1997).

${ }^{26}$ P. Allongue, C. Henry de Villeneuve, S. Morin, R. Boukherroub, and D. D. M. Wayner, Electrochim. Acta 45, 4591 (2000); M. L. Munford, R. Cortès, and P. Allongue, Sens. Mater. 13, 259 (2001).

${ }^{27}$ H. Tolentino, A. Y. Ramos, M. C. M. Alves, R. A. Barrea, E. Tamura, J. C. Cezar, and N. Watanabe, J. Synchrotron Radiat. 8, 1040 (2001).

${ }^{28}$ T. Ressler, J. Phys. IV 7, C2-269 (1997).

${ }^{29}$ C. M. Fu, P. C. Kao, M. S. Tsai, H. S. Hsu, C. C. Yu, and J. C. A. Huang, J. Magn. Magn. Mater. 239, 17 (2002).

${ }^{30}$ J. Geshev, L. G. Pereira, and J. E. Schmidt, Phys. Rev. B 64, 184411 (2001); 66, 134432 (2002).

${ }^{31}$ D. Berling, P. Bertoncini, M. C. Hanf, A. Mehdaoui, C. Pirri, P. Wetzel, G. Gewinner, and B. Loegel, J. Magn. Magn. Mater. 212, 323 (2000). 